\title{
Avaliação e comparação do nível de atividade física entre escolares de 11 a 14 anos de idade
}

\author{
Evaluation and comparison of physical activity level between \\ schools from 11 to 14 years of age
}

\author{
Rosane Bezerra Oliveira' \\ Vanessa de Souza Bulhões ${ }^{1}$ \\ José Robertto Zaffalon Júnior ${ }^{1}$
}

\section{RESUMO}

A prática de atividade física é fundamental para o desenvolvimento saudável da criança, pois o sedentarismo prejudica a saúde, desencadeando problemas na adolescência e vida adulta. Objetivo: analisar o nível de atividade física de crianças de $6^{\circ}$ e $7^{\circ}$ ano de escolas públicas e privadas de Altamira-PA. Métodos: Participaram do estudo 457 alunos de 11 a 14 anos de idade. A coleta de dados foi realizada através do questionário PAQ-A, que é uma versão do IPAQ e foram analisados por meio da estatística descritiva. Resultados: De acordo com os dados encontrados, $50,6 \%$ dos alunos foram considerados pouco ativos e $43,4 \%$ moderadamente ativos; na comparação por sexo, 37,9\% dos meninos são pouco ativos, 50,8\% moderadamente ativos e $10,3 \%$ muito ativo, as meninas $60,9 \%$ são pouco ativas e $37,4 \%$ moderadamente ativas; na comparação por instituição, 52\% dos alunos das escolas pública são pouco ativos e 41,6\% moderadamente ativos, nas escolas particulares $35,9 \%$ são pouco ativos e $60,5 \%$ moderadamente ativos; entre os alunos do $6^{\circ}$ ano $46,5 \%$ são pouco ativos e 48,2\% moderadamente ativos; no $7^{\circ}$ ano 55\% são pouco ativos, 38,2\% moderadamente ativos. Conclusão: Com base nos resultados, percebe-se um maior número de crianças sedentárias em ralação as ativas, e principalmente as meninas que se mostraram menos ativas que os meninos. Por tanto fica evidente a necessidade de incentivo a prática de atividade física para crianças e adolescentes com o intuito de aumentar o nível da prática regular de atividade física.

\section{PALAVRAS-CHAVE}

Atividade física; Estilo de vida sedentário; Criança.

'Universidade do Estado do Pará. 


\section{ABSTRACT}

The physical activity is essential for the healthy development of the child because the sedentary lifestyle affect health, causing problems in adolescence and adulthood. Objective: analyze the level of physical activity of children aged 6 and 7 years of public and private schools from Altamira-PA. Methods: The study included 457 students from 11 to 14 years old. Data collection was performed using the PAQ-A questionnaire, which is a version of the IPAQ and were analyzed using descriptive statistics. Results: According to the findings, $50.06 \%$ of students were considered less active and $43.4 \%$ moderately active; compared by gender, $37.9 \%$ are less active, $50.8 \%$ moderately active and $10.3 \%$ very active, the $60.9 \%$ girls are less active and $37.4 \%$ moderately active; on a per institution, $52 \%$ of students in public schools are very active and $41.6 \%$ moderately active, $35.9 \%$ in private schools are less active and $60.5 \%$ moderately active; among the students of the 6th year $46.5 \%$ are very active and $48.2 \%$ moderately active; the 7 th year $55.1 \%$ are less active, $38.2 \%$ moderately active. Conclusion: Based on the results, it is evident the need to encourage physical activity for children and adolescents in order to increase the level of regular physical activity.

\section{KEYWORDS}

physical activity; sedentary lifestyle; child. 


\section{INTRODUÇÃO}

O sedentarismo pode ser definido como a diminuição ou falta da atividade física regular. É uma condição que resulta em pouco gasto calórico durante o desenvolver de atividades cotidianas, por ser um dos sintomas mais agravantes para a saúde do ser humano. É ainda uma das principais causas do acometimento de doenças como hipertensão arterial, diabetes, ansiedade, aumento do colesterol, como também a obesidade.

Haja visto estes fatores genéticos possuírem forte influência, o sobrepeso e obesidade são principalmente consequências do estilo de vida, como os hábitos alimentares e o nível de atividade física. Por isso os hábitos alimentares saudáveis e a prática de atividade física devem ser recomendados como forma de prevenção desse mal desde a infância, uma vez que crianças obesas estão mais suscetíveis a se tornarem adultos obesos.

A prática de atividade física na infância é fundamental para o desenvolvimento saudável da criança, pois o sedentarismo prejudica a saúde, podendo desencadear problemas na adolescência e até mesmo na vida adulta, e de acordo com Silva e Costa Júnior (2011, p. 42), "a prática regular de atividade física tem sido apontada como um fator relacionado funcionalmente à promoção da saúde dos indivíduos e à prevenção de algumas condições de risco a doenças". Faria, Canabrava e Amorim (2013), também afirmam que a atividade física na infância é importante no desenvolvimento corporal, crescimento e saúde, sendo, portanto, fator fundamental na prevenção de doenças relacionadas ao sedentarismo.

Silva e Costa Júnior (2011), apontam ainda que os jovens e crianças devem estar informados dos benefícios (a curto e longo prazo) da prática regular de atividade física para a sua saúde. Este fator irá contribuir com a motivação da criança em praticar atividade física regularmente. Os autores afirmam ainda que a criança precisa se sentir atraída pela atividade proposta e esse é um desafio a ser superado pelas famílias, escolas e comunidade criando um ambiente que atenda às necessidades das mesmas e incentivando-as.

Com o fácil acesso à tecnologia, nota-se o aumento do comportamento sedentário do ser humano, fazendo com que as crianças despendam muito mais tempo para as atividades de menor gasto calórico em detrimento às práticas de atividade que proporcionam maior queima de calorias. Silva e Costa Júnior (2011, p. 47), apontam que "atualmente parece haver grande valorização e investimento em formas sedentárias de divertimento e um menor envolvimento de jovens com atividades físicas ao ar livre".

As doenças crônicas não transmissíveis (DCNT's) em 2008 eram a causa de $63 \%$ das mortes no mundo, seguindo essa tendência, no Brasil em 2013 foram responsáveis por $72,6 \%$ das mortes, se apresentando com um novo desafio para os gestores em saúde (BRASIL, 2014). Essas DCNT's são resultantes de diversos fatores modificáveis como tabagismo, consumo nocivo de álcool, inatividade física e alimentação não saudável. De acordo com Castro Júnior et al. (2012, p. 956) "A prática de atividade física influencia na qualidade de vida dos indivíduos de forma direta, e, ao mesmo tempo, reduz gastos aos cofres públicos e configura-se como um importante artifício da saúde pública".

É de responsabilidade da saúde pública atuar no combate ao sedentarismo, promovendo campanhas de incentivo à prática regular de atividade física, orientação sobre os riscos que o mesmo causa a saúde da população. Ao considerar principalmente as crianças, por estarem em fase de desenvolvimento, a falta da prática regular de atividade física pode acarretar em obesidade e consecutivamente ao desenvolvimento de outras doenças associadas ao sedentarismo.

Mattos e Neira (2000, p.70), definem a obesidade como o "acúmulo excessivo de gordura corporal, podendo ser causada por alta ingestão alimentar ou baixa atividade física". E ainda, a obesidade é uma doença que se não tratada, pode gerar diversos problemas de saúde devido ao excesso de gordura corporal e a falta da prática de atividade física.

Galvani et al. (2013), afirmam que a inatividade física atrelada a alimentação não saudável contribui de forma significativa para o aumento da obesidade na população em geral, sendo também a causa do desenvolvimento de algumas doenças crônicas. Atualmente as crianças têm maior acesso a alimentos ricos em calorias, sódio, gordura e açúcares, o que 
pode contribuir diretamente para o sobrepeso e obesidade. Segundo Ribeiro et al. (2011), a obesidade é um problema que tem aumentando bruscamente desde a infância, devido a falta da prática regular de atividade física diária.

Saba (2011), ao relatar sobre o sedentarismo infantil, também afirma que crianças e adolescentes com inatividade física costumam levar para a maturidade os costumes da infância, se tornando adultos inativos, pois as crianças têm maior necessidade de atividade física regular para se desenvolver com saúde. Portanto, hábitos saudáveis, alimentação equilibrada e prática regular de atividade física é de grande relevância para que a criança cresça com saúde, pois muitos dos problemas de saúde que são causados pelo sedentarismo só vão se manifestar na vida adulta.

De acordo com Silva e Costa Júnior (2011), a prática diária de atividade física proporciona diversos benefícios para a saúde da criança, contribuindo diretamente na prevenção de doenças crônicas na vida adulta. A prática regular de atividade física desde a infância é fundamental para aquisição de uma meIhor qualidade de vida e um bom desenvolvimento das habilidades motoras, possibilitando futuros adultos ativos. É recomendado uma hora por dia de atividade moderada ou intensa para uma boa qualidade de vida, porém é necessário incentivo e novas estratégias para que as crianças e jovens se sintam atraídas a prática de atividade física diária (FARIA; CANABRAVA; AMORIM, 2013).

$\mathrm{Na}$ infância e na adolescência a obesidade e o sobrepeso têm se tornado um problema grave, pois filhos de pais obesos apresentam maior chance de se tornarem obesos quando crescerem, se comparadas aos filhos de pais eutróficos (MACHADO, 2011). Assim, a prática regular de atividade física das crianças, vários outros fatores são influenciados pelos hábitos dos pais, ou seja, pais sedentários, filhos sedentários. Nesta perspectiva "atividade física é toda ação humana que envolva movimentação e acelere os batimentos cardíacos acima da frequência de repouso" (SABA, 2011, p.52), enquanto o sedentarismo, doença que pode ser tratada no dia a dia, com mudanças simples no estilo de vida, como andar de bicicleta, brincar de pega, fazer caminhadas, jogar futebol entre outras práticas que deveriam sem corriqueiras principalmente na infância.

A infância é a fase em que ocorre várias transformações tanto corporal quanto motora, e nessa fase os hábitos adquiridos possuem grandes chances de serem desenvolvidos ao longo da vida. Incentivar hábitos saudáveis para as crianças é a melhor forma de ter uma vida futura mais saudável. Nesta fase da vida, a melhor forma de prevenir doenças bem como criar hábitos saudáveis está na prática de atividade física. O período mais favorável para a aquisição de hábitos de vida ativos é considerado a infância, à medida que as crianças são inseridas no mundo da atividade física regular, elas aprendem mais sobre seus corpos além de contribuir com a prevenção de fatores de riscos para a saúde (CHEROBIN; PALMA, 2014).

$A$ atividade física regular na infância é importante para o desenvolvimento corporal com um todo, pois proporciona melhor qualidade de vida e mais saúde ao indivíduo. A adoção de um estilo de vida saudável requer que indivíduos mantenham hábitos que promovam a saúde e também a prevenção de doenças ao longo da sua vida. Por isso incentivar a prática regular da atividade física na infância é importante pois isto lhe proporcionará uma vida adulta mais saudável e com mais qualidade de vida (SILVA; COSTA JÚNIOR, 2011).

Para Mendes, Piccoli e Quevedo (2014), a qualidade de vida é definida como a maneira que os indivíduos percebem sua posição na vida, no contexto da cultura, dos sistemas de valores em que vive, é um conceito que é afetado por fatores como a saúde física e psicológica do indivíduo. Em virtude disso, esse conceito muda de indivíduo para indivíduo, pois não depende somente da saúde física, mas também da relação social, pelas crenças, ou seja, pelo ambiente em que se vive, que é caracterizado por onde e pela forma no qual o homem vive.

Saba (2011), afirma que ser saudável no século XXI tornou-se incomum, pois mesmo que as pessoas não manifestem problemas graves de saúde, podem ser consideradas não saudável se não conseguir desenvolver suas capacidades de maneira plena, capaz de produzir e exercer suas atividades diárias.

A busca pela qualidade de vida está cada vez mais frequente na sociedade, principalmente quando se 
trata de saúde, no entanto, não se vê com tanta ênfase essa preocupação com as crianças. Diante disso a educação física tem um papel de fundamental importância no combate ao sedentarismo e incentivo à prática regular de atividade física.

Nessa perspectiva, a escola possui função importante como incentivadora da prática regular de atividade física por meio da disciplina educação física. Pois como afirmam Carvalho e Marega (2012), difícil é a tarefa de conscientização da população sobre a importância da atividade física regular, tal conscientização deve iniciar na escola nas aulas de educação física, pois essas aulas podem ser a única possibilidade de acesso a prática regular de atividade física que muitas pessoas têm. A prática regular de atividade física, inclusive a praticada na escola, proporciona diversos benefícios à saúde e longevidade, melhorando a postura, flexibilidade, coordenação motora, trofismo como também na redução de patologias relacionadas a inatividade física (HESPANHOL JUNIOR et al., 2011).

A disciplina educação física deve integrar o aluno capacitando-o para usufruir dos jogos, dança, lutas, ginásticas e esportes em benefício da cidadania e qualidade de vida das crianças no período escolar (CONFEF, 2002).

A educação física escolar é fundamental para formação das crianças, já que a atividade física interfere diretamente na formação física e na aquisição de habilidades motoras, e uma criança ativa está menos suscetível a desenvolver doenças causadas pelo sedentarismo.

A educação física é de fundamental importância na formação corporal da criança, por ter uma área de atuação diversificada com vantagens de apresentar várias formas de atividades que podem estar proporcionando um melhor desenvolvimento aos alunos, buscando meios diferentes de introduzi-los nas aulas, deixando interessados em participar da mesma, visando sempre o bem-estar do aluno. Entre as consequências do sedentarismo estão a regressão funcional, hipotrofia, redução da flexibilidade e mobilidade articular causando também a obesidade e sobrepeso, possibilitando condições a doenças crônicas (HESPANHOL JÚNIOR et al., 2011). Por isso é necessário incentivar as crianças a prática diária de atividade física, o que irá torná-las ativas com menos chance de desenvolver doenças relacionadas ao sedentarismo.

No entanto, a aquisição de todos esses benefícios advindos da atividade física regular na escola depende do professor de educação física, que é o principal orientador dos exercícios e aprendizado do aluno, além de ser comprometido com sua profissão assim como será possibilitado esse conhecimento. Conforme Faria, Canabrava e Amorim (2013, p. 91), "na escola, as aulas de educação física têm sido a forma tradicional de promoção de Atividade Física regular e orientada".

De acordo com Cherobin e Palma (2014), a prática regular de atividade física na escola exerce influência positiva sobre a saúde e o bem-estar dos indivíduos, sendo comparada aos cuidados de saúde primários. São muitos os relatos referentes os benefícios proporcionados pela prática da atividade física regular na saúde das pessoas, independente se é praticado como lazer ou aquisição de saúde, podendo também em caso de escolares, melhorar no aprendizado não só na educação física, mas também em outras disciplinas curriculares.

Estudos evidenciam que as principais causas do sedentarismo infantil estão ligadas às tecnologias, o aumento da insegurança nas cidades, e a diminuição dos espaços para prática de atividades físicas. Devido a isso, os pais preferem deixar seus filhos em casa jogando vídeo game do que correr risco nas ruas da cidade ou em praças e clubes que não ofereça segurança.

Levando em consideração as consequências que o baixo nível de atividade física regular pode causar na vida das crianças, consecutivamente a adolescência por estarem em fase de transição da infância para ela, por consequência virão os impactos na vida adulta. A falta de informação dos pais da importância de praticar atividade física, tempo para a prática de esporte, ausência de espaços adequado nos bairros, tem levado as crianças a utilizar seu tempo livre com atividades que envolvam tecnologias que levam ao ócio, sendo assim surgiu o interesse de analisar o nível de atividade física das crianças de $6^{\circ}$ e $7^{\circ}$ ano das escolas públicas e privadas. 


\section{PROCEDIMENTOS METODOLÓGICOS}

O presente estudo trata-se de uma pesquisa de campo realizada por meio de levantamento de dados que segundo Gil (1999), é caracterizada pela interrogação direta dos indivíduos que se deseja conhecer, buscando informação do problema estudado, com intuito de coletar resultados precisos, através de procedimento sem dor ou desconforto aos participantes. A mesma tem objetivo descritivo que para Gil (1999), esse tipo de pesquisa tem como base a descrição das características de determinada população, fenômeno ou estabelecimento de relações entre variáveis. A pesquisa é de caráter quanti-qualitativo que de acordo com Teixeira (2010), a quantitativa parte da teoria que engloba a tentativa de explicar algum aspecto da realidade, formulando hipóteses pelo uso de dados, utilizando a linguagem matemática para descrever os fenômenos e suas variáveis. Já a pesquisa qualitativa parte da teoria de compreensão e interpretação de fenômenos, reduzindo a distância entre teoria e dados, contexto e ação.

A pesquisa foi desenvolvida, em 14 escolas públicas e 3 privadas que possuem as séries finais do ensino fundamental do município de Altamira-PA, localizadas no perímetro urbano.

Fizeram parte da pesquisa 457 alunos, sendo 207 meninos e 250 meninas do $6^{\circ}$ e $7^{\circ}$ ano do ensino fundamental, com idade entre 11 e 14 anos de ambos os gêneros. Foram selecionados $10 \%$ dos alunos ( $6^{\circ}$ e $7^{\circ}$ anos) do município de Altamira, conforme o método probabilístico, que segundo Marconi e Lakatos (2003), baseia-se na escolha aleatória do público em que todos os membros da população têm as mesmas chances de ser escolhidos, este processo de amostragem permite a utilização de tratamento estatístico o que compensa os erros amostrais e outros aspectos relevantes para a representação da população.

A escolha do público alvo se deu por estas crianças estarem em fase de transição da infância para a adolescência, o que pode levar à diminuição da prática de atividade física, já que nessa fase começa a surgir diversos interesses que podem tirar o foco da prática regular de atividade física, e por ser um público acessível a aplicação de questionário pois há pressuposição de que todos têm domínio da leitura e uma melhor interpretação de texto, o que pode diminuir a interferência dos pesquisadores.

A coleta de dados foi realizada através do questionário PAQ-A, que é uma versão do IPAQ, validado por Guedes e Guedes (2015), o qual foi desenvolvido para acessar os níveis comuns da prática de atividade física de crianças e adolescentes. O PAQ-A é composto por nove perguntas fechadas com cinco alternativas com o objetivo de classificar o nível de atividade física das crianças e adolescentes, levando em consideração apenas a semana anterior a aplicação do questionário. Desta maneira, incluindo atividades praticadas como esporte, dança ou jogos no qual tenha um dispêndio calórico acima do considerado normal, realizado no ambiente escolar e momento de lazer. Os questionários foram avaliados utilizando-se os dados da própria amostra, no qual foi estabelecido os valores do $1^{\circ}, 2^{\circ}$ e $3^{\circ}$ quartil. Os sujeitos que apresentaram pontuação inferior ao $1^{\circ}$ quartil (percentil 25) foram considerados "muito pouco ativos"; os que apresentaram pontuação entre o $1^{\circ}$ e o $2^{\circ}$ quartil (percentil 50 ou mediana) foram considerados "pouco ativos"; os que apresentaram pontuação entre o $2^{\circ}$ e o $3^{\circ}$ quartil (percentil 75) foram considerados "moderadamente ativos"; e os que apresentaram pontuação superior ao $3^{\circ}$ quartil foram considerados "muito ativos". Os dados foram processados no SPSS 20.

Os resultados obtidos foram analisados por meio da estatística descritiva que de acordo com Gil (1999), ocorre com a descrição dos dados e avalição das generalizações obtidas nos dados coletados, e apresentados em tabela.

A pesquisa respeitou todos os aspectos éticos citados pela resolução $466 / 12$, todos os participantes por serem menor de idade, assinaram o Termo de Assentimento Livre Esclarecido (TALE) e o seu responsável legal assinou o Termo de Consentimento Livre e Esclarecido (TCLE), definindo os procedimentos livres esclarecidos para que os participantes tivessem conhecimento do que iria acontecer. O presente trabalho foi aprovado pelo comitê de ética da Universidade do Estado do Pará, Campus XII Santarém, e estáregistradosoboCAAEn55781816.0.0000.5168. 


\section{RESULTADOS E DISCUSSÃO}

Na Tabela 1 encontram-se os valores obtidos do nível de atividade física dos alunos que fizeram parte do estudo. Entre os resultados, foram analisadas as seguintes categorias: geral, masculino, feminino, escola pública, escola particular, $6^{\circ}$ ano e $7^{\circ}$ ano.

Tabela 1 - Classificação do nível de atividade física.

\begin{tabular}{|c|c|c|c|c|}
\hline Variáveis & Muito pouco ativos & Pouco ativos & $\begin{array}{c}\text { Moderadamente } \\
\text { ativos }\end{array}$ & Muito ativos \\
\hline Geral & $0,7 \%$ & $50,6 \%$ & $43,4 \%$ & $5,3 \%$ \\
\hline Masculino & $1,0 \%$ & $37,9 \%$ & $50,8 \%$ & $10,3 \%$ \\
\hline Feminino & $0,4 \%$ & $60,9 \%$ & $37,4 \%$ & $1,3 \%$ \\
\hline Escola Pública & $0,8 \%$ & $52 \%$ & $41,6 \%$ & $5,6 \%$ \\
\hline Escola Particular & $0 \%$ & $35,9 \%$ & $61,5 \%$ & $2,6 \%$ \\
\hline $6^{\circ}$ ano & $0,9 \%$ & $46,5 \%$ & $48,2 \%$ & $4,4 \%$ \\
\hline $7^{\circ}$ ano & $0,5 \%$ & $55 \%$ & $38,2 \%$ & $6,3 \%$ \\
\hline
\end{tabular}

Fonte: elaborado pelos autores.

Ao analisar os dados gerais, foi percebido na tabela 1, que $50,06 \%$ dos alunos são considerados pouco ativos, prevalecendo sobre as outras classificações, sendo assim a maior parte das crianças e adolescentes praticam pouca atividade física. Moreira (2014, p.13), relata que "Embora se saiba da importância da prática da atividade física na infância e adolescência a evidencia do seu decréscimo com o passar dos anos na fase escolar, mais acentuado na transição entre a fase da criança para a adolescência". Com isso a falta de atividade física nessa faze da vida pode desencadear em problemas de saúde futuros, conforme diz Orsano et al., (2010), mesmo que elas não apresentem sintomas de doenças crônicas degenerativas nesta fase da vida, a manutenção desse nível de atividade física no decorrer dos anos pode acarretar em diversas doenças.

No entanto $43,4 \%$ dos alunos foram classificados como moderadamente ativos, sabe-se que a prática da atividade física é benéfica em todas as fases da vida, pois crianças ativas apresentam maior chance de serem adolescentes ativos, que também têm maior probabilidade de serem adultos ativos (BIELEMANN; XAVIER; GIGANTE, 2014).

Conforme Guedes e Guedes (2015), a atividade física praticada de maneira adequada e suficiente na infância e adolescência pode prevenir ou postergar o aparecimento de disfunções crônico-degenerativas na fase adulta. Tendo em vista que o sedentarismo apresenta consequências evidentes na vida dos indivíduos, principalmente na vida adulta, não obstantes fatores como excesso de peso, obesidade são consequência do mesmo.

Orsano et al. (2011), afirmam que o estilo de vida sedentário acompanhado de comportamentos de risco, são considerados determinantes para comprometer a vida de adultos de ambos os gêneros, pois esses hábitos estabelecidos na infância e adolescência tendem a permanecer até a idade adulta e consequentemente indivíduos inativos com sobrepeso, obesos e outros agravos à saúde.

Ao analisar por sexo, observou-se que $37,9 \%$ dos meninos são pouco ativos, 50,8\% moderadamente ativos e 10,3\% muito ativo, já as meninas $60,9 \%$ são 
pouco ativas, 37,4\% moderadamente ativos e apenas $1,3 \%$ muito ativas, notou-se então uma diferença significativa no nível de atividade física em relação ao sexo masculino e feminino, na qual os meninos têm uma maior prevalência na prática de atividade física. Essa condição também foi percebida em outros estudos realizados com estudantes (COLEDAN et al., 2014; RIBEIRO et al., 2013; FARIA, CANABRAVA e AMORIM 2013; CASTRO JÚNIOR et al 2013).

$\mathrm{O}$ alto índice de sedentarismo entre as meninas pode estar relacionado a menor competência atlética, como afirmam Coledan et al. (2014). Ribeiro et al. (2013), relatam que apesar do aumento da participação das meninas em atividade física e esportiva ainda existe diferença entre meninos e meninas.

No estudo feito por Faria, Canabrava e Amorim (2013), os autores encontraram que uma das possíveis causas de as meninas possuírem menor nível de atividade física regular, pode estar relacionado ao aumento da temperatura pois as participantes relataram que evitam participar de algumas atividades para evitar ou amenizar a sudorese após o intervalo.

Santos (2013), também afirma que as meninas tendem a diminuir a prática de atividade física com o passar da idade. No estudo de Castro Júnior et al. (2013), realizado com estudantes de medicina, os autores encontraram condições semelhantes relacionada aos jovens universitários sendo as mulheres mais sedentárias $(63,1 \%)$ em ralação aos homens $(45,7 \%)$.

Por tanto é possível perceber que a prevalência do sedentarismo em mulheres também é recorrente na idade adulta, o que pode ser consequência dos hábitos da infância e adolescência. É preciso levar em consideração que a infância é a fase mais favorável para aquisição de hábitos de vida saudável, hábitos esses que seguem por toda a vida, corroboram com a firmação (SILVA; COSTA JÚNIOR, 2011, CHEROBIN; PALMA, 2014).

$\mathrm{Na}$ análise por instituição, os alunos de escolas públicas apresentaram que $52 \%$ dos alunos são pouco ativos e 41,6\% moderadamente ativos, enquanto os alunos de escolas particulares $35,9 \%$ são pouco ativos e 60,5\% moderadamente ativos. Os resultados comprovam que os indivíduos das instituições particulares são mais ativos comparados as instituições públicas. Saba (2011), afirma que o sedentarismo deixou de ser privilégio das classes sociais com maior poder aquisitivo, como já foi no passado. Mesmo existindo diferença no nível de atividade física entre os alunos das instituições pesquisadas, sabe-se que a escola é uma grande intermediadora do conhecimento, possuindo grande influência na vida dos escolares, pois ao promover experiências adquiridas neste meio, estará contribuindo diretamente para o desenvolvimento de hábitos de prática regular de atividade física.

Neste sentido Faria, Canabrava e Amorim (2013), enfatizam que o ambiente escolar tem sido um setor chave na promoção de Atividade Física diária para os jovens. Considerando que as crianças passam uma parte substancial do seu dia na escola, a educação física também tem fundamental importância, uma vez que contribui para o aumento do nível de atividade física das crianças e adolescentes, influenciando diretamente na saúde dos mesmos, desta maneira o conhecimento teórico deve ser aliado ao conhecimento prático desenvolvidos nas aulas, para que as crianças e adolescentes tenham mais interesse por essas práticas.

$\mathrm{Na}$ análise por série, entre os alunos do $6^{\circ}$ ano $46,5 \%$ foram classificados como pouco ativos e $48,2 \%$ moderadamente ativos; no $7^{\circ}$ ano $55 \%$ foram classificados como pouco ativos, 38,2\% moderadamente ativos e 6,3\% muito ativos. Constatou-se que no $6^{\circ}$ ano não houve diferença significativa entre pouco ativos e moderadamente ativos, com valores bem semelhantes.

No entanto o $7^{\circ}$ ano apresentou uma diferença relevante entre esses níveis, mostrando um valor significativo de crianças pouco ativas, isso pode se dar pelo fato de eles estarem passando da infância para a adolescência, momento em que começam a surgir interesses por atividades sedentárias, desinteresse pelas brincadeiras, bem como a pela prática de esportes que tenham um maior gasto calórico, dando preferência ao ócio, uso de celulares, televisão, computador, internet, redes sociais e outras tecnologias.

Santos (2013, p.16), corrobora ao dizer que "as crianças e adolescentes estão a cada ano menos ativas e as condições atuais levam essa população a ficarem em casa assistindo televisão, jogando vídeo game e passando horas na internet". Santos (2013), 
relata ainda que são vários os alertas chamando a atenção para o problema da falta de oportunidade que as crianças e jovens de hoje encontram para atingir o nível de atividade física suficiente, mesmo nas atividades escolares ou de forma espontânea fora da escola, para proporcionar uma melhor qualidade de vida desses indivíduos beneficiando sua saúde.

Com isso a disciplina de Educação Física pode contribuir para melhorar o nível de atividade física dessas crianças, pois muitas delas fazem atividade física apenas nas aulas, o que não é suficiente para deixarem de ser pouco ativas. Moreira (2014, p.14), impera que "para a grande parte dos jovens, a educação física escolar passou a ser o único momento em que realizam atividades física planejadas, acompanhadas e avaliadas por um profissional da área". O profissional de Educação Física em suas aulas tem um papel de grande importância como incentivador da prática regular de exercícios conforme afirma Andrade, Figueira Júnior e Miranda (2014), pois a disciplina tem o potencial de atingir a maior parte das crianças e adolescentes para a prática de atividade física.

Em suma as aulas de educação física, o autor reforça que experiências nessas aulas proporcionam oportunidades de envolvimento em exercício físico e consecutivamente melhorias nas habilidades motoras, à medida em que proporciona o aumento do nível de atividade física e contribuindo no comportamento ativo na vida adulta. Já que a disciplina de Educação Física possui uma grade curricular com variedades de conteúdo, pode por meio dessa diversidade tornar as aulas mais atrativas estimulando o interesse das crianças em participar da mesma e consequentemente criando hábitos de vida ativo, pois através dessas vivencias pode surgi o interesse de praticar essas atividades ou esportes desenvolvidos nas aulas em seu momento de lazer.

\section{CONSIDERAÇÕES FINAIS}

Os resultados analisados neste estudo apontam que $50,6 \%$ dos indivíduos participantes da pesquisa foram considerados pouco ativos, ou seja, a maioria dessas crianças e adolescentes não praticam atividade física regularmente o suficiente para um desenvolvimento saudável e com melhor qualidade de vi- da, portanto é preciso conscientizar as crianças sobre sua condição sedentária, para que as mesma tenham ciência das consequências que o sedentarismo pode causar no futuro a sua saúde, pois as crianças sedentárias de hoje tem maiores chances de se tornarem adultos sedentários e consequentemente com problemas de saúde causados pela inatividade.

Com base nos resultados fica evidente a necessidade de incentivo à prática de atividade física para crianças e adolescentes, e principalmente com relação as meninas que se mostraram menos ativas que os meninos, com o intuito de minimizar o sedentarismo, o que irá contribuir na aquisição de hábitos saudáveis para uma vida adulta e na prevenção de doenças crônicas não transmissíveis.

Portanto, faz-se necessário a realização de mais estudos nessa temática para investigar o que leva essas crianças e adolescentes a terem hábitos de vida sedentários, especialmente as meninas que se mostrou menos ativas, com o intuito de conscientizar os mesmos das consequências que o sedentarismo pode causar a seu desenvolvimento saudável e as problemáticas que podem surgir na vida adulta.

\section{REFERÊNCIAS}

ANDRADE, E.I.; FIGUEIRA JÚNIOR, A.; MIRANDA, M.I.j. Ambiente Escolar e Atividade Física: Análise das Variáveis e Instrumentos Utilizados em Estudos na América do Norte e Europa. Revista Brasileira de Ciência e Movimento, São Paulo, v. 22, n. 1, p.157-167, 30 mar. 2014. http://dx.doi. org/10.18511/0103-1716/rbcm.v22n1p157-167.

BIELEMANN, Renata Moraes; XAVIER, Mariana Otero; GIGANTE, Denise Petrucci. Preferência por comportamentos favoráveis à prática de atividade física e nível de atividade física de crianças de uma cidade do sul do Brasil. Ciência e Saúde Coletiva, [s.I.], v. 19, n. 7, p.2287-2296, jul. 2014. FapUNIFESP (SciELO). http://dx.doi. org/10.1590/1413-81232014197.11202013. Disponível em:< http://www.scielo.br/scielo.php?pi$\mathrm{d}=$ S1413-81232014000702287\&script=sci_abstract\&tIn$\mathrm{g}=\mathrm{pt}>$. Acesso em: 03 out. 2016.

BRASIL. Portal da Saúde. Sistema Único de Saúde. Vigilância das doenças crônicas não transmissíveis. 2014. Disponível em: <http://portalsaude.saude.gov.br/index.php/o-ministerio/principal/leia-mais-o-ministerio/671-secretaria-svs/vigilancia-de-a-a-z/doencas-cronicas-nao-transmissiveis/14125-vigilancia-das-doencas-cronicas-nao-transmissiveis>. Acesso em: 28 out. 2016. 
CARVALHO, José Antônio Maluf de; MAREGA, Marcio. Manual de Atividades Físicas para Prevenção de Doenças. Rio de Janeiro: Elsevier, 2012. 1 v.

CASTRO JÚNIOR, Euton Freitas de et al. Avaliação do nível de atividade física e fatores associados em estudantes de medicina de fortaleza-CE. Revista Brasileira de Ciências e Esporte, Florianópolis, v. 34, n. 4, p.955-967, out. 2012. Semestral. Disponível em: <http://www.scielo.br/scielo. php? pid=S0101-32892012000400011 \&script = sci_ abstract\&tlng=pt>. Acesso em: 03 out. 2016.

CHEROBIN, Inaê Angélica; PALMA, Míriam Stock. Perfil de atividade física de escolares provenientes de diferentes redes de ensino e cidades do Rio Grande do Sul. Revista Brasileira de Ciência e Movimento, Rio Grande do Sul, v. 22, n. 4, p.77-87, set. 2014. Disponível em:< https://portalrevistas.ucb.br/index.php/RBCM/article/view/4700>. Acesso em: 03 out. 2016

COLEDAM, Diogo Henrique Constantino et al. Prática esportiva e participação nas aulas de educação física: fatores associados em estudantes de Londrina, Paraná, Brasil. Cadernos de Saúde Pública, [s.I.], v. 30, n. 3, p.533-545, mar. 2014. FapUNIFESP SciELO. http://dx.doi.org/10.1590/0102-311x00087413. Disponível em: <http://www.scielo.br/

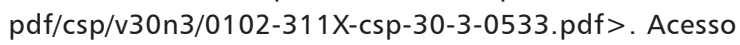
em: 03 out. 2016.

CONFEF, Conselho Federal de Educação Física. REVISTA EDUCAÇÃO FíSICA Nº 05 - Dezembro de 2002. Disponível em: http://www.confef.org.br/extra/revistaef/show. asp?id=3457 acesso em: 05 de junho de 2015.

FARIA, Fernanda R.; CANABRAVA, Karina R.; AMORIM, Paulo R. Nível de atividade física durante o recreio escolar em escola pública e particular.

Revista Brasileira de Ciência e Movimento, Viçosa, v. 1, n. 21, p.90-97, 23 jan. 2013. Disponível em: < http://portalrevistas.ucb.br/index.php/RBCM/article/view/3223/2473>. Acesso em: 02 nov. 2015.

GALVANI, Carlos A. et al. Aptidão física aeróbica em crianças e sua relação com obesidade e componentes da síndrome metabólica. Revista Brasileira de Ciência e Movimento, Abc Paulista, v. 1, n. 21, p.123-131, 15 mar. 2013. Disponível em: < http://portalrevistas.ucb.br/index.php/RBCM/ article/view/3869/2478>. Acesso em: 03 nov. 2015.

GIL, Antonio Carlos. Métodos e técnicas de pesquisa social. 5. Ed. São Paulo: Editora Atlas S. a, 1999. p.207.

GUEDES, Dartagnan Pinto; GUEDES, Joana Elisabete Ribeiro Pinto. Medida da atividade física em jovens brasileiros: reprodutibilidade e validade do paq-c e do paq-a. Revista Brasileira de Medicina do Esporte, [s.I.], v. 21, n. 6, p.425432, dez. 2015. Fap UNIFESP (SciELO). http://dx.doi. org/10.1590/1517-869220152106147594
GUEDES, Nirla Gomes; LOPES, Marcos Venícios de Oliveira. EXERCÍCIO FÍSICO EM PORTADORES DE HIPERTENSÃO ARTERIAL. Revista Gaúcha de Enfermagem, Porto Alegre (rs), v. 31, n. 2, p.367-374, jun. 2010. Disponível em: <http:// www.scielo.br/scielo.php?script $=$ sci_arttext $\&$ pid =S1983-14472010000200023 >. Acesso em: 11 out. 2015.

HESPANHOL JUNIOR, Luiz C. et al. Efeito do método Isostretching na flexibilidade e nível de atividade física em indivíduos sedentários saudáveis. Revista Brasileira de Ciência e Movimento, São Paulo, v. 1, n. 19, p.26-31, 20 maio 2011. Disponível em: <http://portalrevistas.ucb.br/index.php/RBCM/article/view/1671/1848>. Acesso em: 03 nov. 2015.

MACHADO, Yara Líbia. Sedentarismo e suas Consequências em Crianças e Adolescentes. 2011. 52 f. Tese (Bacharel) - Curso de Educação Física, Instituto Federal de Educação Ciências e Tecnologia Sul de Minas - Campus Muzambinho Bacharelado em Educação Física, Muzambinho, 2011. Disponível em: <http://200.131.11.44/attachments/1681_17.pdf>. Acesso em: 04 jun. 2015.

MARCONI, Marina de Andrade; LAKATOS, Eva Maria. Fundamentos de Metodologia Científica. 5. ed. São Paulo: Editora Atlas S.a., 2003. 310 p.

MATTOS, Mauro Gomes de; NEIRA, Marcos Garcia. Educação Física na Adolescência: construindo o conhecimento na escola. São Paulo: Phorte Editora, 2000. 144 p.

MENDES, Daisiane; PICCOLI, João Carlos Jaccottet; QUEVEDO, Daniela Müller de. Qualidade de vida relacionada à saúde de escolares do ensino fundamental de Campo Bom, RS. Revista Brasileira de Ciência e Movimento, Campo Bom, v. 4, n. 22, p.47-54, abr. 2014. Disponível em: < http:// portalrevistas.ucb.br/index.php/RBCM/article/ view/4902/3492 >. Acesso em: 02 nov. 2015.

MOREIRA, Rodrigo Baptista. Nível de atividade física nas aulas de educação física. 2014. 100 f. Tese (Doutorado) Curso de Educação Física. Universidade Federal de Rio Grande do Sul. Porto Alegre, 2014. Disponível em: <http://www. lume.ufrgs.br/bitstream/handle/10183/97842/000921176. pdf? sequence $=1>$. Acesso em: 09 out. 2016 .

ORSANO, Vania Silva Macedo et al. Estilo de vida e níveis de aptidão física relacionada à saúde em adolescentes de Demerval Lobão/PI. Revista Brasileira de Ciência e Movimento, Demerval Lobão, PI, v. 18, n. 4, p.81-89, out. 2010. Disponível em: <https://portalrevistas.ucb.br/index.php/ RBCM/article/view/2419>. Acesso em: 03 out. 2016.

RIBEIRO, Ariovaldo de Souza et al. Prevalência de sobrepeso e obesidade em crianças de $3^{\mathrm{a}}$ e $4^{\mathrm{a}}$ série do ensino fundamental. Revistas Unoeste, São Paulo, v. 03, n. 1, p.01-05, jan. 2011. Disponível em: < http://revistas.unoeste.br/revistas/ojs/index.php/cv/article/viewFile/558/462> . Acesso em: 18 jun. 2015. 
RIBEIRO, José Antônio Bicca et al. Nível e importância atribuídos à prática de atividade física por estudantes do ensino fundamental de uma escola pública de pelotas/ rs. Revista Mackenzie de Educação Física e Esporte, Pelotas, v. 12, n. 2, p.13-25, 2013. Semestral. Disponível em:< http://editorarevistas.mackenzie.br/index.php/remef/article/view/3791/4772>. Acesso em: 03 out. 2016.

SABA, Fabio. Mexa-se: atividade física, saúde e bem-estar. $3^{a}$ edição Revisada. São Paulo: Phorte Editora, 2011. 328 p.

SANTOS, Melissa dos. Desempenho das habilidades motora na infância e predição dos níveis de atividade física ao longo do tempo. 2013. 79 f. Dissertação (Mestrado) Curso de Educação Física, Pedagogia do Movimento, Esco- la de Educação Física e Esporte, Universidade de São Paulo, São Paulo, 2013. Disponível em: <http://www.teses.usp.br/ teses/disponiveis/39/39133/tde-23052013-165048/pt-br. php >. Acesso em: 03 out. 2016.

SILVA, Paulo Vinícius Carvalho; COSTA JUNIOR, Áderson Luiz. Efeitos da atividade física para a saúde de crianças e adolescentes. Psicologia Argumento, Curitiba, v. 29, n. 69, p.41-50 janeiro/março 2011. Disponível em: <http:// www2.pucpr.br/reol/index.php/PA?dd1 $=4525 \& d d 99=-$ view>. Acesso em: 05 jun. 2015.

TEIXEIRA, Elizabeth. As três metodologias: Acadêmica, da ciência e da pesquisa. $7^{\circ}$ ed. Petrópolis, Rj: Editora Vozes, 2010. 203 p. 